\title{
Discovery of a double ring in the core of $\eta$ Carinae $^{\star}$
}

\author{
S. Hony ${ }^{1}$, C. Dominik ${ }^{1}$, L. B. F. M. Waters ${ }^{1,2}$, V. Icke ${ }^{3}$, G. Mellema ${ }^{3}$, R. van Boekel ${ }^{4,1}$, A. de Koter ${ }^{1}$, \\ P. M. Morris ${ }^{5}$, M. Barlow ${ }^{6}$, P. Cox ${ }^{7}$, and H. U. Käuff ${ }^{4}$ \\ 1 Astronomical Institute "Anton Pannekoek", University of Amsterdam, Kruislaan 403, 1098 SJ Amsterdam, \\ The Netherlands \\ 2 Instituut voor Sterrenkunde, Katholieke Universiteit Leuven, Celestijnenlaan 200B, 3001 Heverlee, Belgium \\ 3 Sterrewacht Leiden, Postbus 9513, 2300 RA Leiden, The Netherlands \\ 4 European Southern Observatory, Karl-Schwarzschild Strasse 2, 85748 Garching, Germany \\ 5 SIRTF Science Center, Caltech, Mail Code 314-6, 1200 East California Boulevard, Pasadena, CA 91125, USA \\ 6 University College London, Department of Physics and Astronomy, Gower Street, WC1E 6BT London, UK \\ 7 Institut d'Astrophysique Spatiale, Bât. 121, Université de Paris Sud, 91405 Orsay Cedex, France
}

Received 14 May 2001 / Accepted 21 August 2001

\begin{abstract}
We report the discovery of a double ring structure in the waist of the nebula surrounding $\eta$ Carinae. The rings are detected in the mid-IR dust continuum at wavelengths of 7.9, 11.9, 12.9 and $20 \mu \mathrm{m}$. The dust in the rings has a temperature of about $300 \mathrm{~K}$. The orientation of the rings is inclined with respect to the axis of the homunculus by either 37 or 58 degrees. The central star is not in the projected centre of the structure defined by the two rings. This geometry is reminiscent of that seen in SN1987A and some planetary nebulae. We discuss several possible origins for this remarkable geometry and its orientation.
\end{abstract}

Key words. circumstellar matter - stars: emission-line - stars: $\eta$ Car - stars: massive - infrared: stars

\section{Introduction}

The star $\eta$ Car is one of the best studied, but still poorly understood members of the class of Luminous Blue Variables (Davidson \& Humphreys 1997). $\eta$ Car shows a spectacular, strongly bipolar nebula referred to as the homunculus (e.g. Morse et al. 1998), that was created during the great eruption of $1840-1860$. The homunculus is sometimes described as a hollow flask expanding with velocities between 400 and $\sim 800 \mathrm{~km} \mathrm{~s}^{-1}$. A conspicuous equatorial skirt is seen in the Hubble Space Telescope (HST) images. Proper motion studies of the condensations in the skirt show that these were ejected during the great eruption (Morse et al. 2001). There is strong evidence that $\eta$ Car is a binary star with a period of 5.52 years and orbital eccentricity of 0.6 (Damineli 1996; Damineli et al. 2000). The X-ray light curve of $\eta$ Car is consistent with a colliding winds model (Corcoran et al. 2001), and supports the binary model for $\eta$ Car.

Many studies have focused on infrared imaging of the dusty homunculus (e.g. Gehrz et al. 1973; Smith et al. 1998; Polomski et al. 1999, hereafter Pol99; Gehrz et al. 1999; Pantin \& Le Mignant 2000, hereafter PL00). These

Send offprint requests to: S. Hony, e-mail: hony@astro.uva.nl

* Based on observations taken at the European Southern Observatory, La Silla, Chile. studies show that at near-IR wavelengths the central star dominates while the lobes are faint. Note that speckle polarimetry of the core of $\eta$ Car indicates that the central star is not viewed directly but is obscured (Falcke et al. 1996). At mid-IR wavelengths several equatorial emission blobs northeast and southwest of the central star are visible. These blobs are interpreted as evidence for an equatorial torus (Pol99; Morris et al. 1999, hereafter Mor99). PL00 show that the bipolar lobes cannot be empty and suggest the presence of a second shell interior to the optically visible homunculus. HST observations also point to an inner structure (Ishibashi et al. 2000).

The Infrared Space Observatory (ISO) spectrum of $\eta$ Car (taken Jan. 1996) shows three spectral components: a power law between 2 and about $10 \mu \mathrm{m}$, arising from the central point source, a $T \sim 250 \mathrm{~K}$ component, attributed to the lobes, and a $T \sim 110-130 \mathrm{~K}$ component, attributed to a cold, $\sim 15 M_{\odot}$ equatorial torus (Mor99). It was suggested that the massive equatorial torus was present before the great eruption and caused the highly bipolar shape of the nebula.

In this Letter we discuss new mid-IR images of $\eta$ Car that resolve the equatorial blobs and show that they have a highly symmetric ring-like shape with a surprising orientation with respect to the homunculus. The images may 


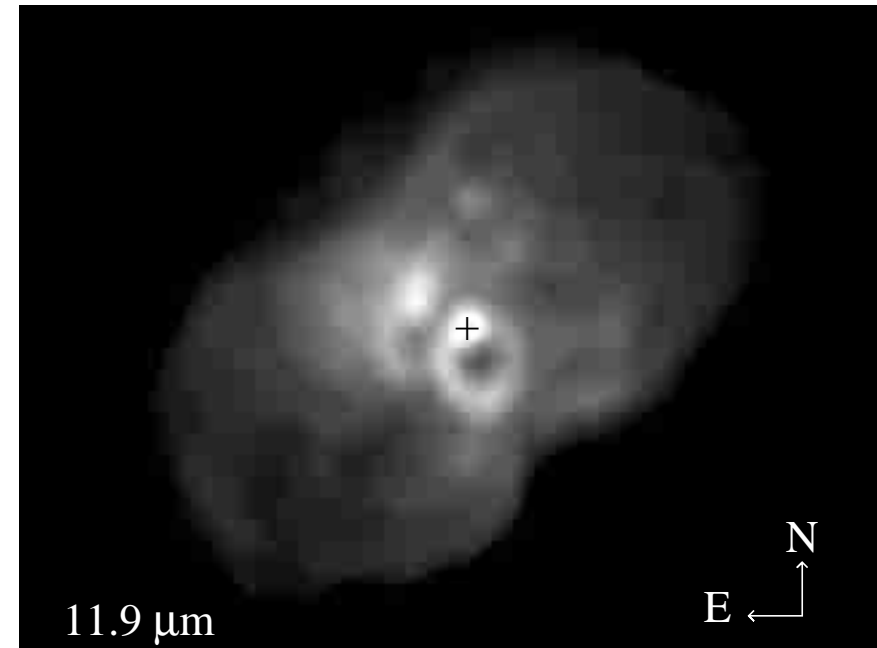

Fig. 1. TIMMI2 image of $\eta$ Car in the $11.9 \mu \mathrm{m}$ narrow band filter. We applied a square root function to enhance the intensity contrast. The "+" indicates the central source.

point to a drastic change in the orientation of the plane of symmetry of the system some time after the great eruption.

\section{The observations}

We observed $\eta$ Car between 3 and 6 March 2001, with the TIMMI2 (Reimann et al. 2000; Käufl et al. 2000) mid-IR camera attached to the ESO $3.6 \mathrm{~m}$ telescope at La Silla, Chile. The camera is equipped with a $320 \times 240$ pixel array; we applied a pixel scale of $0.2 \mathrm{arcsec} /$ pixel in both the $N$ and the $Q$ band. We used a chop throw of $22 \operatorname{arcsec}$ north-south and a nod of 30 arcsec east-west. This allowed for both the chopped as well as the nodded positions to fall onto the detector. In the final images, we combined the positive and negative images resulting from the chopping and nodding positions. $\eta$ Car was observed in the $M$ band $(4.8 \mu \mathrm{m})$, at several wavelengths in the $N$ band using narrow-band filters $(7.9,11.9,12.9 \mu \mathrm{m})$, and in the $Q$ band $(20 \mu \mathrm{m})$. The observing conditions were variable with a high humidity and seeing ranging between 0.3 and about 1 arcsec. Apart from the images, a number of longslit $N$-band spectra covering the entire nebula were taken, as well as a few $Q$-band spectra. In this paper, we concentrate on the 7.9, 11.9, 12.9 and $20 \mu \mathrm{m}$ images. We will report on the other images, as well as on the spectroscopy in a subsequent study.

The images were reduced using a shift-and-add technique, where the shift between individual frames was determined from a least-squares comparison between the images. The resulting images were then deconvolved using an empirically determined point spread function obtained by observing $\gamma$ Cru with the same set-up as $\eta$ Car. For the $Q$ band image we have no good empirical point spread function. However, given that in the $Q$ band TIMMI2 at the $3.6 \mathrm{~m}$ is diffraction-limited, we have adopted a Gaussian beam of 1.25 arcsec to deconvolve the $Q$ band image.

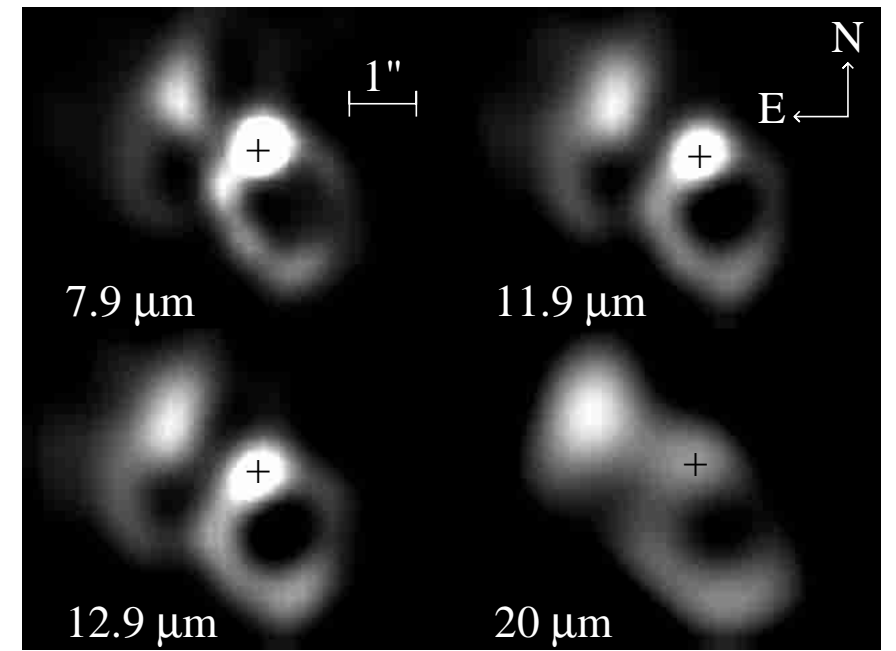

Fig. 2. Multi wavelength observations of the central part of the $\eta$ Car nebula. The double ring structure is traced at all four wavelengths. The "+" indicates the central source.

We show the final images in Figs. 1 and 2. Figure 3 shows a temperature map, derived assuming that the total flux in the images is equal to that seen in the ISO-SWS spectrum (Mor99). We realize that the variability of $\eta \mathrm{Car}$ may introduce errors in this calibration. However, we verified the calibration at 7.9 and $11.9 \mu \mathrm{m}$ using $\gamma$ Cru and found values of $1.6 \times 10^{4}$ and $5.2 \times 10^{4} \mathrm{Jy}$. The agreement between the two methods is within 10-20 per cent. We stress that unless the shape of the spectrum of $\eta$ Car has changed considerably between 1996 and 2001, our method should result in reasonable estimates of the temperature. Note that the strong $10 \mu \mathrm{m}$ silicate band (e.g. Mor99) may introduce some errors $(\sim 15 \%)$ in the temperature map.

\section{Description of the images}

The mid-IR image presented in Fig. 1 shows the familiar homunculus with an overall shape which is similar to that reported by previous authors (e.g. Morse et al. 1998; Smith et al. 1998; Mor99; PL00). At the core of the homunculus we find a central point-like source which dominates the emission from the nebula at short wavelengths. This point source very likely coincides with the central star or binary. Mor99 show that the 2-8 $\mu \mathrm{m}$ wavelength range of the ISO spectrum of $\eta$ Car is well represented by a power law, with a steeply rising spectrum towards longer wavelengths. It is therefore reasonable to assume that the central point source is responsible for the power law component. At $20 \mu \mathrm{m}$ the central point source no longer dominates the emission, which shows that there is not a large amount of cold dust at this location. If the continuum in the $2-8 \mu \mathrm{m}$ region is due to thermal emission from dust, the power law nature of the spectrum points to a flat temperature gradient in the hottest dust (nearest to the central object), which is typically seen in optically thick disks. We stress that imaging at the sub-arcsecond scale is required to determine the true geometry of the innermost regions 


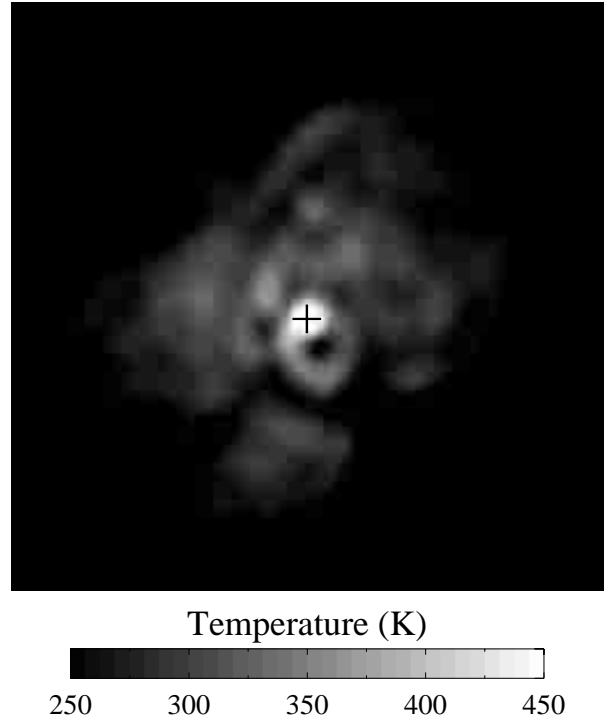

Fig. 3. Temperature map as derived from the 7.9, 11.9, 12.9 and $20 \mu \mathrm{m}$ observations (see text). The central point source, indicated by the "+", has a derived temperature of $600 \mathrm{~K}$.

of the $\eta$ Car nebula. Optical speckle imaging polarimetry supports the presence of a disk (Falcke et al. 1996).

The $N$ band and $Q$ band images give a detailed view of the innermost few arcsec of the homunculus, where in the optical images the waist is situated. Previous studies revealed the presence of emission blobs of different intensity roughly 1.5 arcsec northeast and southwest of the central point source. These emission blobs have been interpreted as evidence for an equatorial torus (Pol99; Mor99). This torus has been held responsible for the strongly bipolar geometry of the homunculus (Mor99). From the ISO spectrum a temperature of the matter in the torus of 110 to $130 \mathrm{~K}$ was derived, and a dust mass of about $0.15 M_{\odot}$.

Our new images show for the first time the detailed geometry of the material emitting at these wavelengths down to very low brightness levels. The images show that the mid-IR emission is not due to limb-brightening of a torodial dust distribution seen edge-on (in Mor99 assumed to be co-spatial with the distribution of the massive cold material). Rather, the blobs reported by previous studies turn out to be two arcs of emission. A careful inspection of the $11.9 \mu \mathrm{m}$ image shows that the arc southwest of the central star is a closed ring. We will refer to these two structures as "the two rings" in what follows, because both arcs have the same size and inclination. The southwest ring passes through the central point source, so this point source cannot be at the centre of the rings. The northeast structure has an irregular surface intensity with a strong intensity maximum in the north. The axis connecting both rings is not aligned with the projection of the long axis of the homunculus on the sky (see below). We note that one of the two rings can be recognised in the $20 \mu \mathrm{m}$ images published by Pol99; Mor99 and PL00. However, the factor of two better spatial resolution at $10 \mu \mathrm{m}$ compared to
$20 \mu \mathrm{m}$ together with the high sensitivity allows for a much clearer view of these structures.

The temperature map shows that the two rings have roughly similar temperatures of $280-380 \mathrm{~K}$. These temperatures agree well with the values derived by previous studies (Smith et al. 1998; Pol99; PL00). If we assume that there is no large difference in the foreground extinction towards both rings, this shows that the dust in the two rings is heated similarly. The question arises what the location of the 110-130 K massive dust component, inferred from the ISO observations is. Since this spectral component peaks at $30 \mu \mathrm{m}$ and no flux jumps due to SWS aperture transitions are seen, it must fit within the SWS band $3 \mathrm{~A}$ beam. The low temperature implies a different physical component from that in the lobes or rings, such as a torus, as previously suggested by Mor99. Davidson \& Smith (2000) have shown that this cold component should have a minumum projected area of $37 \operatorname{arcsec}^{2}$ in order to reproduce the required flux levels. An inclined torus of that size (which would not show limb-brightening) fits easily within the SWS beam.

We have constructed a geometrical model for the midIR emission of the equatorial regions (sketched in Fig. 4). The model assumes the presence of two circular rings. We varied the position angle on the sky as well as the inclination angle until a good match with the observations was obtained. The result is shown in Fig. 4. We find that the rings have a diameter of 1.8 arcsec and a de-projected distance between the two rings of 3 arcsec. The structure is rotated by 117 or 297 degrees in the plane of the sky with respect to north-south (depending on which of the two rings is in front). The inclination between the major axis of the homunculus and of the axis connecting the centres of the two rings is either 37 or 58 degrees, using an inclination of the axis of the homunculus to the plane of the sky of 40 degrees $^{1}$. The error on these angles is about 10 to 15 degrees, mostly determined by the wide range of values given in the literature for the inclination of the homunculus (e.g. Hillier \& Allen 1992). Note that the region in our model where the projection of both rings overlap on the sky coincides with an intensity maximum in the observed images, again supporting our two ring model.

\section{Discussion}

The new mid-IR images reveal a more complicated structure of the inner few arcsec of the homunculus' equatorial regions than previously suggested. The images show that two highly symmetric ring-like structures are present, as well as an intensity maximum towards the northeast. We find that the axis connecting the centre of the two rings is inclined with respect to the major axis of the homunculus by either 37 or 58 degrees, depending on which

${ }^{1}$ The angle $(\delta)$ between the major axis of the homunculus $(\mathrm{H})$ and the axis connecting the centres of the rings $(\mathrm{R})$ is: $\cos \delta=\left(\sin \theta_{\mathrm{H}} \sin \theta_{\mathrm{R}}+\cos \theta_{\mathrm{H}} \cos \theta_{\mathrm{R}}\right) \sin i_{\mathrm{H}} \sin i_{\mathrm{R}}+\cos i_{\mathrm{H}} \cos i_{\mathrm{R}}$, where $i$ is the inclination and $\theta$ the position angle. 


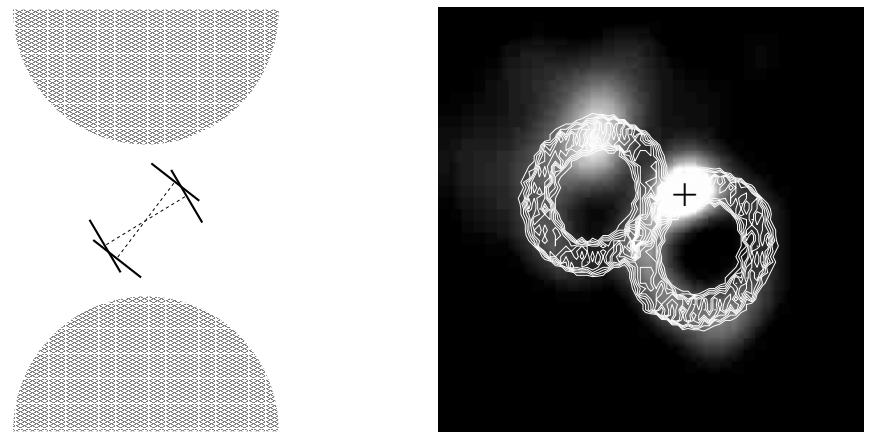

Fig. 4. Simple geometrical model. The left panel sketches the position of the rings viewed from above the plane defined by the main axis of the homunculus and the line through the centers of the rings. There are two solutions because in the projection seen from Earth it is unknown which ring is in front. The right panel shows the projected rings overlaid on the $11.9 \mu \mathrm{m}$ image.

of the two rings is in front, and assuming an inclination for the homunculus with respect to the plane of the sky of 40 degrees. Kinematics of the gas in the rings is needed to decide which of these two possibilities is correct.

PL00 show that the inner homunculus structure seen at $20 \mu \mathrm{m}$ represents regions of increased column density. If indeed the rings trace a density enhancement, then they could be denser rings in a bipolar nebula, similar to the rings in SN1987A (Burrowset al. 1995). We also note that this geometry shows strong resemblance to that seen in PNe such as He2-113 (Sahai et al. 2000) and Hb 12 (Welch et al. 1999) including the two rings, the misalignment between the bipolar structure and the rings, and the offset of the central star with respect to the ring structure. It seems reasonable to conclude that the physical mechanism causing these structures is generic and acts in high mass as well as in low mass objects.

A number of mechanisms to produce double rings in bipolar nebulae have been proposed for PNe (Icke 1988) and SN1987A (Crotts \& Heathcote 2000), but which one applies where has not been established. In all cases the rings are perpendicular to the major axis of the nebula, which for $\eta$ Car implies that the major axis of this inner nebula is at a significant angle (37 or 58 degrees) to the major axis of the homunculus.

It seems difficult to avoid the conclusion that there must have been a change in the orientation of the outflow between the moment of production of the homunculus and the creation of the double ringed structure. This strongly favours the binary model for the $\eta$ Car system. The shredded appearance of the skirt in the HST images and the proper motion of the condensations indicate that the equatorial regions were highly perturbed by the great eruption. It is therefore likely that the rings were produced after the great eruption. 1) The change of orientation could result from an asymmetry in the mass loss during the great eruption. 2) It could be due to tidal interaction of the eccentric binary with material in its environment. The required mass for such a process can be estimated in the following crude way. A gravitational perturbation can act most easily in the apocenter. In a Keplerian motion about a mass $M_{*}$ with eccentricity $e$ and semi-major axis $a$, the apocenter distance and velocity are given by $r=a(1+e)$ and $v^{2}=\frac{G M_{*}(1-e)}{a(1+e)}$. The required acceleration to change the orbital inclination by about 1 radian is of the order of $v^{2} / a(1+e)$. Since the great eruption of 1840 , about $N=160 / 5.5 \approx 29$ orbital periods have passed. In order to produce the required total change in $N$ steps, a disturbing mass $\tilde{M}$ at distance $R$ would have to fulfil the condition $\frac{\tilde{M}}{M_{*}}\left(\frac{a}{R}\right)^{2}=\frac{1}{N} \frac{1-e}{(1+e)^{2}} \approx 0.0054$, with $M_{*}$ the reduced mass of the binary. Therefore with $e=0.6$ and $R \approx a$, then $\tilde{M} \approx 5 \times 10^{-3} M_{*}$. A moderate amount of mass close to the binary could already be sufficient to explain the observed change in the system orientation.

Acknowledgements. We thank N. Ageorges for the excellent help with data-acquisition. VI thanks A. van Genderen for discussions. This work was supported by a NWO Pionier grant to LBFMW and a NWO Spinoza grant to E. P. J. van den Heuvel.

\section{References}

Burrows, C. J., Krist, J., Hester, J. J., et al. 1995, ApJ, 452, 680

Corcoran, M. F., Ishibashi, K., Swank, J. H., \& Petre, R. 2001, ApJ, 547, 1034

Crotts, A. P. S., \& Heathcote, S. R. 2000, ApJ, 528, 426

Damineli, A. 1996, ApJ, 460, L49

Damineli, A., Kaufer, A., Wolf, B., et al. 2000, ApJ, 528, L101

Davidson, K., \& Humphreys, R. M. 1997, ARA\&A, 35, 1

Davidson, K., \& Smith, N. 2000, Nature, 405, 532

Falcke, H., Davidson, K., Hofmann, K.-H., \& Weigelt, G. 1996, A\&A, 306, L17

Gehrz, R. D., Ney, E. P., Becklin, E. E., \& Neugebauer, G. 1973, Astrophys. Lett., 13, 89

Gehrz, R. D., Smith, N., \& Krautter, J. 1999, in Eta Carinae at The Millennium, ASP Conf. Ser., 179, 20

Hillier, D. J., \& Allen, D. A. 1992, A\&A, 262, 153

Icke, V. 1988, A\&A, 202, 177

Ishibashi, K., Gull, T. R., Davidson, K., \& STIS ID Team 2000 , Amer. Astron. Soc. Meet., 197, 0814

Käufl, H. U., Ageorges, N., Dietzsch, E., et al. 2000, The Messenger, 102, 4

Morris, P. W., Waters, L. B. F. M., Barlow, M. J., et al. 1999 , Nature, 402, 502

Morse, J. A., Davidson, K., Bally, J., et al. 1998, AJ, 116, 2443

Morse, J. A., Kellogg, J. R., Bally, J., et al. 2001, ApJ, 548, L207

Pantin, E., \& Le Mignant, D. 2000, A\&A, 355, 155

Polomski, E. F., Telesco, C. M., Piña, R. K., \& Fisher, R. S. 1999, AJ, 118, 2369

Reimann, H., Linz, H., Wagner, R., et al. 2000, Proc. SPIE, 4008, 1132

Sahai, R., Nyman, L. ., \& Wootten, A. 2000, ApJ, 543, 880

Smith, N., Gehrz, R. D., \& Krautter, J. 1998, AJ, 116, 1332

Welch, C. A., Frank, A., Pipher, J. L., Forrest, W. J., \& Woodward, C. E. 1999, ApJ, 522, L69 